SHS Web of Conferences 21, 03004 (2015)

DOI: $10.1051 /$ shsconf/20152103004

(C) Owned by the authors, published by EDP Sciences, 2015

\title{
Construire une conception scientifique du vivant avec des élèves de 5-7 ans: approche didactique pour mieux comprendre les processus d'apprentissage et les enjeux développementaux
}

\author{
Céline Grancher ${ }^{1, a}$, Yann Lhoste ${ }^{1,2}$ et Patricia Schneeberger ${ }^{1,2}$ \\ ${ }^{1}$ Équipe E3D, LACES (EA4140), Université de Bordeaux, France \\ ${ }^{2}$ ESPE d'Aquitaine, 49 rue de l'École Normale, 33021 Bordeaux Cedex, France
}

\begin{abstract}
Résumé. Cet article s'intéresse au vivant en tant qu'objet de savoir et aux phénomènes d'enseignement-apprentissage de ce concept au début de l'école primaire en France (élèves de 5-7 ans, classes de CP-CE1). Nous nous interrogeons sur les contenus à enseigner et les situations possibles à mettre en œuvre dans les classes pour que des élèves construisent une conception scientifique du vivant. Nous délimitons d'abord le concept de vivant dans une perspective d'enseignement à de jeunes élèves. Puis nous identifions un des enjeux de l'enseignement-apprentissage du vivant: permettre aux élèves de construire un autre rapport au vivant que celui de la vie de tous les jours. Nous abordons les situations scolaires comme des processus d'acculturation scientifique scolaire. Les élèves sont en effet incités à entrer dans une culture scientifique en s'appropriant à la fois des savoirs relatifs au vivant et des pratiques de la biologie. À partir de deux séquences d'enseignement réalisées en CP puis en CE1 avec les mêmes élèves, nous repérons des indicateurs qui témoignent de la construction d'une conception scientifique du vivant par les élèves. Nous montrons également que les processus d'acculturation scientifique dans lesquels les élèves sont engagés ont des effets sur leur développement.
\end{abstract}

\begin{abstract}
This paper focuses on the concept of life and how it can be taught in early elementary school in France (5-7 years old). We study scientific knowledge to teach and possible classroom sessions that may allow pupils to build scientific approach of life. First, we try to define the concept of life for young pupils. Then, we identify the major issue of learning this concept in elementary school: pupils can build a scientific relationship with living things that is different from that of everyday life. Our research consists in observing classroom sessions as scientific acculturation processes. Pupils are encouraged to get involved in scientific culture by taking ownership of scientific knowledge and practice. Two teaching sequences done with the same pupils during two consecutive years of school (grades 1 and 2) are analysed. We identify indicators that reveal change in pupils' ideas of life towards building scientific ideas. We also show that scientific acculturation processes have effects on pupils' development.
\end{abstract}

\footnotetext{
${ }^{a}$ Céline Grancher : celine.grancher@u-bordeaux.fr
} 
Remarque préalable : cet article fait suite à la communication orale effectuée lors du colloque « la vie et le vivant : de nouveaux défis à relever dans l'éducation » en mai 2015 au cours du $83{ }^{\text {ème }}$ congrès de l'ACFAS à Rimouski. Il reprend largement la présentation tout en souhaitant mettre en relation notre travail et les autres contributions du colloque.

\section{Introduction}

Le travail que nous présentons dans cet article consiste à étudier l'entrée d'élèves dans une culture scientifique à l'école primaire. Or selon Bachelard [1], l'accès à cette culture scientifique est une tâche difficile: il s'agit de se détacher de ses opinions et de ses préjugés pour acquérir une connaissance ouverte et dynamique. On mesure alors toute la difficulté à laquelle des enseignants vont être confrontés lorsqu'ils ont pour objectif de faire construire à leurs élèves des savoirs scientifiques, sans figer ces savoirs, mais en engageant les élèves dans des processus dynamiques d'apprentissage. C'est précisément la compréhension de ces processus et leur caractérisation qui est au cœur de cette contribution. Celle-ci s'inscrit dans le volet 3 du colloque, car nous travaillons sur l'enseignement-apprentissage du concept de vivant à l'école primaire française (classes de CP-CE1, élèves âgés de 5 à 7 ans). Notre question centrale est la suivante : comment orienter l'enseignement pour que de jeunes élèves construisent une conception scientifique du vivant en contexte scolaire ? Une première partie interrogera les savoirs relatifs au vivant afin de délimiter ce qu'est une conception scientifique du vivant pour de jeunes élèves. La deuxième partie mettra en avant les enjeux à enseigner le vivant dès l'école primaire et proposera des éléments de cadrage théorique. La troisième partie sera consacrée à la présentation des situations de classe et de la méthodologie de recherche. Enfin, la quatrième partie exposera les résultats à travers des analyses de productions d'élèves et de moments de classe.

\section{Délimitation du concept de vivant pour des élèves de 5-7 ans}

Dans la perspective d'un enseignement du concept de vivant à des jeunes élèves, il nous fallait délimiter les savoirs en jeu et identifier ce que ces élèves pourraient construire comme apprentissages pour aller vers une conceptualisation scientifique du vivant.

\subsection{Les points d'appui}

Qu'est-ce donc qu'une conception scientifique du vivant que des élèves pourraient construire au début de l'école primaire? Nous présentons ici les travaux qui nous ont servi à délimiter le concept de vivant pour des élèves de 5-7 ans.

D'abord, et bien que nous ne leur accordons pas le même statut que les autres sources, nous avons regardé les textes des programmes officiels de l'école primaire (MEN, 2008) [2]. Pour les niveaux qui nous intéressent (classes de CP et CE1, cycle 2), l'étude du concept de vivant appartient au domaine d'activités du monde scolaire " découvrir le monde du vivant». Il est question d'amener les élèves à comprendre ce qui distingue vivant et non vivant puis à leur faire repérer les caractéristiques du vivant ; sont cités la naissance, la croissance, la reproduction, la nutrition et les régimes alimentaires des animaux. On constate que les programmes sont assez succincts quant aux contenus à enseigner et qu'ils ne semblent pas interroger les caractéristiques du vivant : celles citées sont-elles exhaustives ? Suffit-il de posséder une de ces caractéristiques pour être vivant?

Nous avons alors cherché à identifier les savoirs savants relatifs au vivant à travers une analyse épistémologique. Celle-ci nous a permis de nous rendre compte de toute la difficulté à vouloir définir le vivant pour les scientifiques eux-mêmes. Non seulement, il n'y a pas de définition unique du vivant 
(Kostyrka, 2014) [3], mais c'est une pluralité de définitions qui coexistent (cf. différentes contributions à l'ouvrage Comment définir la vie? dirigé par Bersini et Reisse, 2007) [4]. Chaque scientifique selon sa spécialité (biochimie, génétique...) propose une définition de la vie en essayant de dégager ce qui est spécifique au vivant. Certains scientifiques mettent l'accent sur la capacité de reproduction des êtres vivants, d'autres sur celle de synthèse de leur propre matière organique, d'autres encore sur l'organisation de tout système vivant, d'autres enfin sur les interactions d'un être vivant avec son milieu. Morange tranche en affirmant que "la définition de la vie n'est pas à chercher dans une ou quelques caractéristiques qui lui seraient propres, mais dans la réunion et le couplage de ces caractéristiques. " (Morange, 2007, p.69) [5]. Son point de vue converge avec celui développé par Guichard et Deunff : "la vie se définit par un faisceau de caractères, tous constitutifs de la complexité de la vie » (Guichard \& Denuff, 2001, p.30) [6]. Le vivant peut se définir par un ensemble de caractéristiques communes aux êtres vivants (croissance, nutrition, reproduction, mort). En outre, situer ce concept dans une perspective dynamique apparaît important. Tout être vivant est en effet un système ouvert qui échange de la matière, de l'énergie et de l'information avec son milieu. Au delà de la pluralité des approches scientifiques du vivant, (nous renvoyons le lecteur aux articles de M. Dell'Angelo, C. Simard, et M. Coquidé dans cet ouvrage [7, 7, 9] qui rendent compte de façon plus détaillée de l'évolution historique de ce concept), il nous semble qu'une définition scientifique du concept de vivant consiste à délimiter ce qui est propre au vivant, c'est-à-dire les propriétés que les êtres vivants ont en commun, mais qui se manifestent de différentes façons.

Deux remarques s'imposent à ce stade. La première concerne les termes qui entrent en jeu lorsqu'on souhaite définir le concept: vie, vivant, être vivant. Comme le mentionnent Bardel et Triquet (1997) [9], ces termes sont différents et ne recouvrent pas exactement les mêmes acceptions. Il nous semble que la délimitation du concept de vivant ne peut pas se priver des travaux sur la vie, notamment à cause les contours imprécis entre vie et vivant. Il est d'ailleurs probable que les élèves utilisent indifféremment les deux termes dans leurs énoncés. Nous n'y voyons pas de difficulté majeure si ce n'est que nous pensons que le mot vivant, moins utilisé dans le langage de tous les jours, serait plus approprié au cours d'activités scientifiques. La deuxième remarque porte sur la transposition des savoirs savants pour des jeunes élèves. Il ne fait aucun doute que la définition du vivant retenue à l'école est nécessairement adaptée par rapport à celles des scientifiques, ne serait-ce que parce que les élèves de 5-7 ans ne possèdent pas encore les capacités pour raisonner à l'échelle cellulaire par exemple. La formulation du concept de vivant avec eux ne pourra donc pas se faire via l'idée que tout organisme vivant est formé de cellules.

Pour envisager l'enseignement du vivant pour des élèves de 5-7 ans, nous nous sommes également appuyés sur des recherches antérieures en didactique des sciences. Celles-ci permettent d'avoir un aperçu des idées des élèves à propos du vivant et de repérer celles qui peuvent constituer des obstacles à la construction d'une conception scientifique du vivant. De façon générale, les élèves âgés de 5 à 7 ans n'ont pas de difficulté à reconnaître les êtres humains et les animaux communs comme des êtres vivants. Ils considèrent souvent que ce qui bouge est vivant et prennent l'Homme comme modèle du vivant. Guichard et Deunff [6] identifient en l'occurrence que les conceptions dominantes des enfants de 5-7 ans sont l'animisme et l'anthropomorphisme. Ces modes de pensée amènent les élèves à considérer que les éléments naturels tels que le soleil sont vivants parce qu'ils bougent, mais pas les végétaux, car ils ne bougent pas (Nury, Lamarque \& Caron, 1996) [11]. Or le mouvement n'est pas un critère discriminant pour distinguer ce qui vivant de ce qui ne l'est pas. Il peut ainsi constituer un obstacle. De même, le fait que tous les êtres vivants ne ressemblent pas à l'Homme peut aussi constituer un obstacle.

Finalement, qu'est-ce que construire une conception plus scientifique du vivant avec des élèves de 5-7 ans ? Pour nous, c'est leur permettre d'interroger leurs conceptions initiales et de les dépasser pour aller vers des savoirs scientifiques compatibles avec une vision dynamique du vivant; c'est construire ce qui est propre au vivant à travers des pratiques scientifiques telles que l'observation, la comparaison; c'est utiliser une ou plusieurs caractéristiques communes pour reconnaître ce qui est vivant. 


\subsection{Les choix réalisés}

A partir des différents points d'appui précédemment développés, nous avons choisi de travailler autour du concept de vivant selon deux entrées :

- la première approche est centrée sur la distinction vivant - non vivant en adéquation avec les programmes, mais en amenant les élèves à utiliser des caractéristiques scientifiques du vivant pour faire la différence entre vivant et non vivant. Cela implique de ne pas utiliser le mouvement seul, mais de convoquer la croissance, la nutrition, la reproduction et la mort ;

- la deuxième approche consiste à travailler autour d'une propriété commune aux êtres vivants, car les enfants ont parfois du mal à généraliser la vie aux végétaux. Dans le cadre de notre recherche, nous avons décidé de travailler sur la croissance parce que non seulement il est possible de mettre en place des expérimentations avec de jeunes élèves en suivant des plantations et/ou un élevage, mais aussi, et surtout, parce que la croissance peut être mise en lien avec la nutrition et resituée dans le cycle de vie d'un individu où l'on peut alors s'interroger sur l'origine de nouveaux individus et ainsi aborder des questions relatives à la reproduction.

Cette partie montre à quel point le concept de vivant est délicat à délimiter dans une perspective d'enseignement. Nous avons essayé d'expliciter les sources qui nous ont conduits à faire des choix quant aux savoirs enseignés. Mais il est légitime de s'interroger sur les enjeux inhérents à l'enseignement-apprentissage de ce concept dès l'école primaire.

\section{Enjeux de l'enseignement - apprentissage du vivant et positionnements théoriques}

La problématique centrale du colloque est de mettre en lumière l'intérêt à éduquer au vivant à travers la formule «de nouveaux défis pour l'éducation ». Alors même qu'on constate toute la difficulté à circonscrire le concept de vivant, pourquoi vouloir l'enseigner à de jeunes élèves ? Cette question, à l'interface entre sciences et sociétés, a été abordée au cours du colloque, notamment dans les contributions de M.-H. Parizeau, F. Piron et M. Dell'Angelo (dans cet ouvrage) [ 7,12,13].

\subsection{Pourquoi enseigner le vivant si tôt ?}

De façon réductrice, nous pourrions dire qu'enseigner le vivant dès le début de l'école primaire répond à une commande institutionnelle (MEN, 2008) [2]. Mais nous avons vu que les termes des programmes sont relativement flous et n'interrogent pas les savoirs en jeu. L'enjeu majeur serait de faire construire aux élèves un autre rapport au vivant que celui de la vie de tous les jours, un rapport qui soit compatible avec les approches scientifiques actuelles du vivant. Cela s'inscrit dans une perspective bachelardienne [1] des apprentissages selon laquelle une connaissance scientifique objective se construit contre l'expérience première. Comme souligne Dell'Angelo, l'importance est " de dépasser une pensée anthropomorphique, de se décentrer pour comprendre d'autres modes de vie, d'autres besoins. " (Dell'Angelo, 2009, p.17) [15]. Par conséquent, notre parti pris est de considérer que la construction du concept de vivant ne se limite pas à l'acquisition de savoirs scientifiques, mais que ce nouveau rapport au monde participe plus largement à l'entrée dans une culture scientifique et cela nous amène à préciser des éléments de notre cadre théorique.

\subsection{Ancrages théoriques pour étudier les processus d'acculturation scientifique}

En référence d'une part aux travaux de Bruner (1996) [16], qui considère l'éducation comme une entrée dans la culture, et d'autre part aux thèses socio-constructivistes de Vygotski (1934/1997) [17] et aux recherches de didacticiens comme Driver, Asoko, Leach, Scott et Mortimer (1994) [18], nous considérons les situations d'enseignement-apprentissage à l'école comme des moments où les élèves peuvent accéder à une culture scientifique. A ce titre, nous proposons d'étudier les processus 
d'apprentissage à l'œuvre dans une classe comme des processus d'acculturation scientifique scolaire. Le terme d'acculturation, importé de la sociologie (Cuche, 2010) [19], est à comprendre comme entrée dans une culture scientifique. Les élèves vont être amenés à dépasser leurs conceptions issues de leur culture privée, de leurs expériences personnelles pour aller vers des conceptions scientifiques, en adéquation avec le domaine de la biologie. Ils construisent des savoirs scientifiques relatifs au vivant à travers des pratiques scientifiques qui mettent en jeu de nouvelles formes d'activités comme poser des questions ou argumenter une position. Ainsi les processus d'acculturation scientifique scolaire peuvent se définir comme les dynamiques à travers lesquelles des élèves accèdent à une culture scientifique dans le contexte scolaire.

Plus largement, le cadre de cette recherche est celui de la théorie historico-culturelle du psychisme (Vygotski, 1934/1997) [17] s'agissant du développement des concepts chez l'enfant, mais aussi des liens entre les apprentissages scolaires et le langage. Comme le précise Brossard (2013) [20], c'est un véritable travail d'appropriation du monde de la culture scientifique que l'élève doit réaliser afin de réorganiser, transformer les concepts quotidiens qu'il possède et construire de nouveaux concepts scientifiques en prenant appui sur les précédents. Cette appropriation suppose un travail par le langage et nous situons notre contribution dans la lignée des travaux qui ont étudié les liens entre les pratiques langagières et la construction de savoirs en sciences (Jaubert, 2007 ; Schneeberger \& Vérin, 2009) $[21,22]$.

En définitive, notre recherche poursuit trois objectifs :

- trouver des indicateurs qui permettent de repérer que les élèves sont engagés dans des processus d'acculturation scientifique ;

- dégager les conditions favorables pour induire de tels processus en classe ;

- apprécier les effets des apprentissages sur le développement des élèves.

Nous souhaitons en effet placer notre recherche dans une perspective développementale afin de comprendre comment l'appropriation d'une première culture scientifique par des élèves va non seulement les amener à construire des savoirs en sciences, mais va aussi contribuer à la construction de nouvelles formes d'activités du psychisme humain (Brossard, 2013) [20]. Pour cela, nous avons élaboré un dispositif de classe que nous allons présenter dans la partie suivante en explicitant la méthodologie de notre recherche.

\section{Méthodologie de la recherche et présentation du dispositif de classe conçu et mis en œuvre}

\subsection{Orientations retenus pour l'enseignement du vivant et concrétisation dans le dispositif de classe}

Le dispositif de classe a été conçu selon certaines orientations concernant l'enseignement du concept de vivant à de jeunes élèves (voir partie 2.2) et dans le but de générer des processus d'acculturation scientifique. Il intègre donc des contraintes de recherche et c'est la raison pour laquelle il ne s'agit pas de situations ordinaires, mais de situations forcées au sens d'Orange (2010) [23].

Le dispositif consiste à suivre les mêmes élèves pendant les deux premières années de la scolarité élémentaire, en CP et en CE1. Cela doit permettre d'appréhender les effets développementaux des apprentissages scientifiques en jeu. Deux séquences, de découverte du monde du vivant (en cohérence avec les programmes; MEN, 2008) [2] ont été construites en collaboration avec les enseignants des classes. Au total, trois enseignants ont mis en œuvre les séances dans deux classes de CP la première année (en 2014) puis dans deux classes de CE1 l'année suivante (en 2015) à raison d'une séance par semaine pendant 10 à 12 semaines pour chaque séquence. 38 élèves ont participé au projet parmi lesquels 31 ont suivi les deux séquences (les autres ayant changé d'école en cours d'année scolaire).

En CP (figure 1), la séquence avait pour objectif de travailler avec les élèves sur les critères scientifiques qui permettent de distinguer ce qui est vivant de ce qui ne l'est pas. L'orientation retenue correspond à la première approche du vivant que nous avons présentée. 


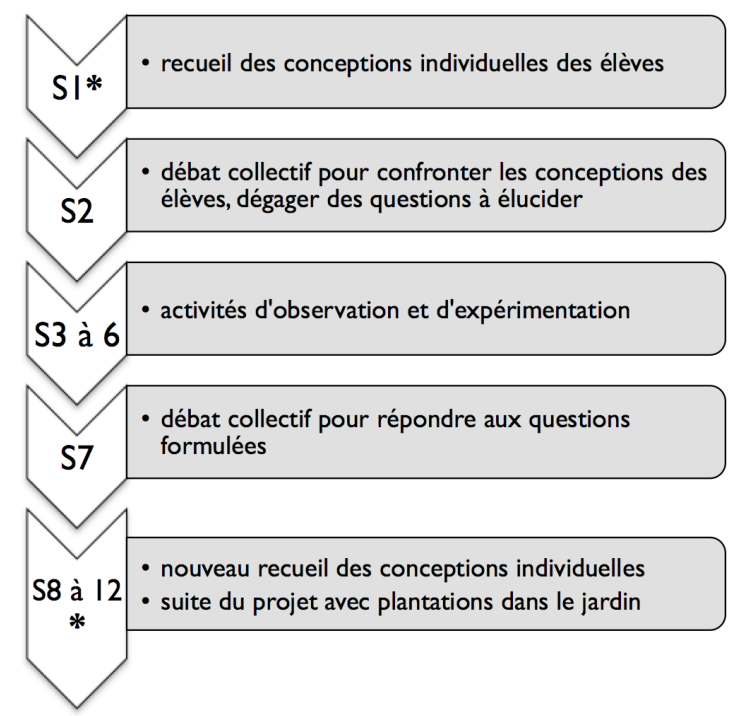

Figure 1. Aperçu de la séquence mise en œuvre en $\mathrm{CP}$, année $\mathrm{N}(\mathrm{S}=$ séance $; *$ = repère pour les moments qui seront traités dans les résultats)

En CE1 (figure 2), le concept de vivant a été abordé selon la deuxième approche, c'est-à-dire en travaillant spécifiquement sur une propriété commune aux êtres vivants. Pour les raisons évoquées précédemment, nous avons décidé de travailler sur la croissance à travers les deux questions suivantes : qu'est-ce que cela veut dire grandir? Comment font les êtres vivants pour grandir?

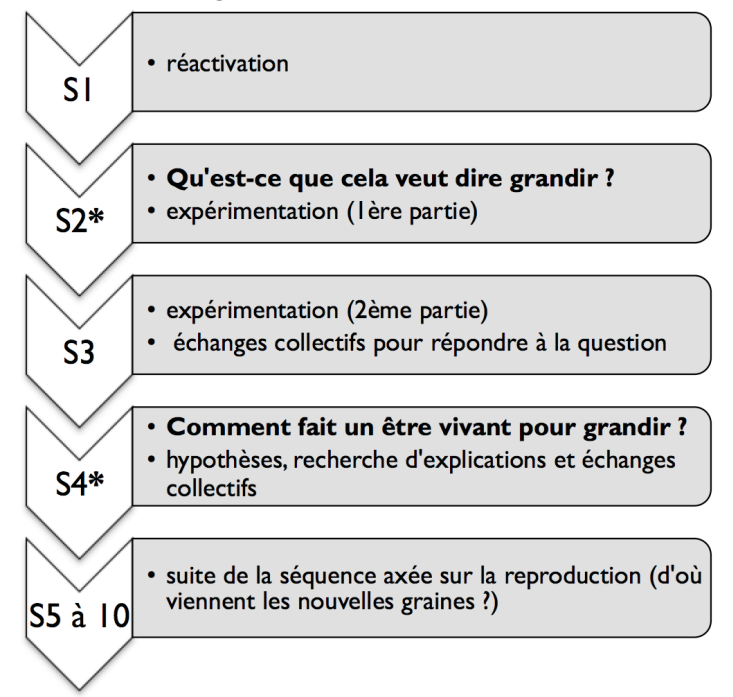

Figure 2. Aperçu de la séquence mise en œuvre en $\mathrm{CE} 1$, année $\mathrm{N}+1\left(\mathrm{~S}=\right.$ séance ${ }^{*}=$ repère pour les moments qui seront traités dans les résultats)

Ces orientations retenues sont différentes de celle de l'enseignement du vivant qu'on rencontre souvent dans les classes de CP et CE1 où non seulement l'étude du vivant se limite à lister des caractéristiques communes aux êtres vivants, mais aussi la démarche d'enseignement n'inclut pas nécessairement l'appropriation de pratiques scientifiques qui participent d'après nous à l'acculturation scientifique au vivant. 


\subsection{Précisions méthodologiques sur le recueil et l'analyse des données}

Les données ont été recueillies dans les classes par les enseignants et par nous. Seules celles utilisées dans cet article seront détaillées. En CP, ce sont des écrits des élèves réalisés lors des séances 1 et 10 (voir figure 1) qui ont été collectés. Les enseignants ont recueilli les conceptions individuelles des élèves en dictée à l'adulte et nous ont transmis une trace de ces écrits. Nous avons parallèlement réalisé un recueil complémentaire auprès de classes témoins de CP n'ayant pas mis en œuvre la séquence. Pour ces écrits, nous avons réalisé nous-mêmes le recueil des conceptions de 24 élèves. En $\mathrm{CE} 1$, nous avons mené des entretiens individuels à l'oral avec 10 élèves, répartis dans les deux classes testées, après la séance 2 (voir figure 2). Les données recueillies sont des enregistrements audio que nous avons retranscrits. La séance 4 a été filmée en classe et nous avons effectué la retranscription des échanges. Des écrits individuels des élèves ont également été collectés au début de la séance 5 .

Notre approche méthodologique s'appuie sur une étude de cas comportant deux séquences successives impliquant les mêmes élèves. Il s'agit pour nous de chercher à expliquer comment ces élèves parviennent à construire une conception scientifique du vivant et en quoi les processus d'acculturation scientifique peuvent provoquer une forme de développement de leur psychisme. Cela devrait nous permettre d'apporter des éléments de réponse à la question centrale de notre recherche, à savoir valider ou non les orientations retenues pour enseigner le vivant à de jeunes élèves.

Nous avons réalisé des analyses qualitatives de type thématiques Paillé et Mucchielli (2012) [24] à partir des énoncés écrits et/ou oraux des élèves et des enseignants. Ces analyses sont combinées à d'autres modalités dont une analyse quantitative des traces écrites des élèves.

\section{Résultats quant à la construction d'une conception scientifique du vivant par les élèves}

Pour rendre compte de l'avancée des élèves dans la construction du concept de vivant, nous proposons de suivre une élève, Nina (prénom modifié), au cours des activités mises en place en CP puis en CE1. Le choix s'est porté sur cette élève, car ses productions écrites et orales révèlent des évolutions importantes dans la façon dont elle conceptualise le vivant. Nous allons nous attacher à montrer dans un premier temps comment les idées de Nina évoluent vers une conception plus scientifique du vivant puis dans un deuxième temps comment cette conception est enrichie.

\subsection{Avancer dans la construction du concept de vivant : une première phase en CP}

\subsubsection{Exemple de Nina à 5 ans $(C P)$}

Nina est une élève ayant un niveau de compétences scolaires satisfaisant pour ce stade de l'année de CP. Lors de la première séance, l'enseignant lui demande de classer des éléments selon ce qu'elle pense et il note en dictée à l'adulte ses explications. Il s'agit d'une activité de tri au sens de Lecointre (2004) [25] dans la mesure où les élèves doivent discriminer ce que vivant et ce qui ne l'est pas. Toutefois, ils sont amenés à regrouper des éléments ayant des caractéristiques communes du vivant, et le fait de regrouper des éléments partageant les mêmes caractéristiques par catégories correspond à l'action de classer. Comme les élèves explicitent leurs choix par des critères divers, nous parlons de classement (l'activité va au delà d'un choix binaire, mais ce n'est pas non plus une classification). Nous présentons ce qu'a fait Nina lors de son premier écrit E1 (voir tableau 1) pour 5 des 10 éléments, car ils permettent de donner un aperçu représentatif des idées de l'élève (les autres éléments étaient un vélo, des nuages, un ordinateur, une fleur et un arbre). Elle classe correctement les enfants, la poule et le caillou. En revanche, elle dit ne pas savoir classer les graines et pense que le soleil est vivant. Ce qui nous renseigne davantage ce sont les explications que Nina fournit pour justifier son classement. Que celui-ci soit valide ou non, on constate que Nina utilise le mouvement (souligné en trait plein) et des références anthropomorphiques (souligné en pointillés), ce qui est en adéquation avec les conceptions dominantes décrites chez les élèves de cet âge (voir partie 2.1). 
Tableau 1. Extrait de la première trace écrite de Nina

\begin{tabular}{|c|c|c|}
\hline \multicolumn{3}{|c|}{$\begin{array}{l}\text { E1 = trace écrite réalisée lors de la } \mathbf{1}^{\mathbf{e}} \text { séance } \\
\qquad \mathrm{V}=\text { Vivant } ; \mathrm{NV}=\text { Non Vivant } ; \mathrm{nsp}=\text { ne sait pas }\end{array}$} \\
\hline éléments & classement & explications données par Nina \\
\hline enfants & $\mathrm{V}$ & parce qu'ils marchent / dès qu'on mange ça nous fait grandir \\
\hline poule & $\mathrm{V}$ & parce qu'elle a des yeux \\
\hline caillou & NV & parce qu'il bouge pas et il parle pas \\
\hline graines & nsp & on le mange, mais je sais pas si elles sont vivantes \\
\hline soleil & $\mathrm{V}$ & parce qu'il bouge \\
\hline
\end{tabular}

Si on compare avec son deuxième écrit E2, réalisé à la fin de la séquence en $\mathrm{CP}$, même si les éléments à classer ont été modifiés (les précédents éléments ont été classés collectivement en classe lors de la séance 7), on remarque que le classement de Nina est complètement valide : cette fois-ci, les graines et la lune sont classées respectivement dans vivant et non vivant. En plus, les explications que donne Nina témoignent d'une évolution de sa façon de reconnaître ce qui est vivant: elle utilise désormais des caractéristiques du vivant (souligné avec des vagues) et surtout elle en fournit plusieurs, en particulier lorsqu'elle convoque le mouvement.

Tableau 2. Extrait de la deuxième trace écrite de Nina

\begin{tabular}{|l|l|l|}
\hline \multicolumn{3}{|c|}{ E2 = trace écrite réalisée lors de la 10 ${ }^{\mathbf{e}}$ séance } \\
\hline éléments & classement & explications données par Nina \\
\hline chat & $\mathrm{V}$ & il peut se nourrir / il peut grandir / il peut se reproduire \\
\hline escargot & $\mathrm{V}$ & parce qu'il peut bouger / il peut se nourrir \\
\hline montagnes & $\mathrm{NV}$ & parce qu'elles grandissent pas / parce qu'elles se nourrissent pas \\
\hline $\begin{array}{l}\text { graines } \\
\text { (autre sorte) }\end{array}$ & $\mathrm{V}$ & parce qu'elles se nourrissent / parce qu'elles peuvent grandir \\
\hline lune & $\mathrm{NV}$ & parce qu'elle ne bouge pas / parce qu'elle ne grandit pas \\
\hline
\end{tabular}

Il y a donc une évolution nette des conceptions chez cette élève, ce qui lui permet de faire la différence entre ce qui est vivant et ce qui ne l'est pas. Cependant ce résultat concerne une seule élève de la classe. C'est la raison pour laquelle nous avons aussi quantifié la tendance observée chez l'ensemble des élèves participant à ce dispositif par rapport à des élèves du même âge n'ayant pas suivi la séquence en $\mathrm{CP}$.

\subsubsection{Résultats quantitatifs relatifs à la construction d'une conception scientifique du vivant dès le CP}

Le tableau 3 rassemble les proportions des productions écrites des élèves qui fournissent un classement valide vivant - non vivant et qui utilisent des caractéristiques scientifiques pour justifier leurs classements. Les traces écrites individuelles et successives sont notées E1, E2 et E2'. Nous avons comptabilisé la quantité d'éléments que les élèves avaient correctement classé dans leurs écrits (colonne 2) et les occurrences des caractéristiques scientifiques du vivant dans l'ensemble des explications des élèves (colonne 3). 
Tableau 3. Comparaison de l'évolution des proportions d'élèves de CP convoquant des caractéristiques scientifiques du vivant pour attribuer la vie à des éléments à classer.

\begin{tabular}{|c|c|c|}
\hline & $\begin{array}{|lr|}\text { proportions } & \text { de } \\
\text { classements } & \text { valides } \\
\text { V/NV } & \\
\end{array}$ & $\begin{array}{l}\text { proportions des énoncés des élèves faisant } \\
\text { apparaître des caractéristiques scientifiques du } \\
\text { vivant }\end{array}$ \\
\hline $\begin{array}{l}\text { E1 } \\
\text { (avant la séquence) }\end{array}$ & $71 \%$ & $5 \%$ \\
\hline $\begin{array}{l}\text { E2 } \\
\text { (après la séquence) }\end{array}$ & $94 \%$ & $63 \%$ \\
\hline $\begin{array}{l}\text { E2' } \\
\text { (classes témoins) }\end{array}$ & $78 \%$ & $13 \%$ \\
\hline
\end{tabular}

La proportion de classements valides vivant - non vivant (noté V/NV), avant tout enseignement, est donnée par l'ensemble de écrits E1. Nous constatons que les classements V/NV de départ sont globalement élevés $(71 \%)$. Cette proportion augmente nettement chez les élèves ayant suivi la séquence $(+23 \%)$ alors que ceux n'ayant pas travaillé spécifiquement sur le concept de vivant n'obtiennent pas des résultats similaires aux élèves testés. Est encore plus remarquable l'évolution des explications des élèves. A travers leurs énoncés, il est possible d'inférer sur leurs conceptions du vivant et on constate que les élèves de $\mathrm{CP}$ ayant participé à la séquence utilisent plus largement des caractéristiques du vivant $(+58 \%)$ alors que ceux des classes témoins, qui ont par ailleurs suivi des enseignements en lien avec le monde vivant librement choisis par leurs enseignants, n'en mobilisent pas autant (seulement 13\% contre 63\%).

Ces résultats sont, selon nous, la trace de processus d'acculturation scientifique : le dispositif de classe a un impact sur l'évolution des conceptions des élèves. Pour aller plus loin dans la compréhension de ces processus d'apprentissage, il serait possible d'analyser les situations intermédiaires, notamment les séances d'échanges collectifs afin de caractériser finement ce qui contribue à transformer durablement les idées des élèves. Mais nous cherchons à comprendre si les choix d'enseignement effectués favorisent la construction par les élèves d'une conception scientifique du vivant. Poursuivons donc la présentation des résultats en CE1 afin de montrer que les évolutions constatées en $\mathrm{CP}$ se retrouvent l'année suivante, et qu'à ce titre, on peut dire qu'elles traduisent la mise en jeu de processus d'acculturation scientifique.

\subsection{Enrichir le concept de vivant : une deuxième phase en CE1}

\subsubsection{Suivi de Nina huit mois plus tard}

Comme mentionné dans la présentation du dispositif, nous avons travaillé avec les mêmes élèves pendant deux ans. Mais avant d'élaborer la deuxième séquence en CE1, un écrit intermédiaire des élèves a été réalisé pour avoir un aperçu de ce dont ils se souvenaient s'agissant du vivant. Voici ce que notre élève Nina a formulé quand nous lui avons demandé de dire avec ses mots qu'est-ce que cela veut dire vivant : "c 'est l'arbre parce qu'il pousse comme nous, c'est la poule parce qu'elle peut bouger, c'est le chat parce qu'il peut faire comme la poule, c'est nous les enfants parce qu'on est vivant, c'est aussi l'ouf parce qu'il y a un petit poussin ». On peut remarquer que Nina définit le vivant à travers des exemples d'êtres vivants. Elle prend pour chacun une caractéristique précise : pour l'arbre la croissance, pour la poule et le chat le mouvement, pour l'œuf l'idée de reproduction. Il ressort que si nous avions proposé aux élèves tels que Nina de travailler à nouveau selon la première approche (distinguer vivant et non vivant), les élèves auraient pu se contenter d'identifier un seul critère distinct pour un être vivant. Or le concept de vivant se construit, selon nous, à travers les propriétés communes aux êtres vivants. On peut donc penser que la deuxième approche amènera les élèves à enrichir leurs conceptions du vivant. 


\subsubsection{Focalisation sur les énoncés de Nina à 6 ans (CE1)}

Si les élèves s'accordaient à dire que tous les êtres vivants grandissent, ils ne formulaient pas clairement ce que cela signifie. Les situations en CE1 ont donc d'abord porté sur la question « qu'estce que cela veut dire grandir? » Les élèves ont suivi trois représentants d'êtres vivants (des enfants, des poules et des plantes) dont ils ont mesuré la taille et la masse à différents moments. A l'issue de ces séances, Nina est capable de formuler les indicateurs qui permettent d'affirmer que les êtres vivants grandissent (en gras dans le tableau 4).

Tableau 4. Extrait 1 : retranscription de l'entretien individuel de Nina ( $\mathrm{M}=$ maîtresse $)$

M : $\quad$ qu'est-ce qu'on pourrait faire pour voir que les êtres humains / les enfants ils grandissent

\begin{tabular}{ll}
\hline Nina : & se mesurer \\
\hline $\mathrm{M}:$ & ah se mesurer / pourquoi c'est intéressant se mesurer \\
\hline Nina : & $\begin{array}{l}\text { parce que on voit que par exemple on a/ on avait / on est mesuré 30 et après } \\
\text { si on se remesure un peu plus tard eh ben / eh ben on va faire 30-31 peut- } \\
\text { être ou plus grand }\end{array}$ \\
\hline $\mathrm{M}:$ & mmh alors ça veut dire quoi quand on passe de 30 à 31 \\
\hline Nina : & on grandit \\
\hline M : & $\begin{array}{l}\text { on grandit d'accord / on augmente de taille on avait dit // est-ce qu'on le voit } \\
\text { avec autre chose qu'on grandit / y a la taille qui devient plus grande et quoi } \\
\text { d'autre / regarde on avait eu une autre idée (indique l'affiche) }\end{array}$ \\
\hline Nina : & se peser \\
\hline : : & se peser / qu'est-ce qu'on fait quand on se pèse \\
\hline Nina : & eh ben on regarde notre poids qu'on a \\
\hline M : & bon alors qu'est-ce qui se passe quand on grandit / ton poids il fait quoi \\
\hline Nina : & i/ i/ il est de plus en plus lourd
\end{tabular}

Ensuite, les situations de classe se sont orientées vers la recherche d'explications en essayant de répondre à la question " comment les êtres vivants grandissent? ». Nous suivons toujours Nina, âgée désormais de 6 ans. Le tableau 5 est un extrait de la suite de l'entretien individuel réalisé après la séance 2. La question «comment les êtres vivants font pour grandir? » est posée pour la première fois.

Tableau 5. Extrait 2 : retranscription de l'entretien individuel de Nina $(M=$ maîtresse $)$

M : donc quand on grandit est-ce qu'on peut dire / on reste pas pareil alors / on est un être vivant on grandit ça veut dire qu'on est capable d'augmenter de taille de se transformer et à ton avis ça vient d'où ce qu'on arrive à fabriquer

\begin{tabular}{|c|c|}
\hline Nina : & euh c'est quand on mange \\
\hline $\mathrm{M}:$ & quand on mange / comment c'est possible \\
\hline $\begin{array}{c}\text { Nina : } \\
{[\ldots]}\end{array}$ & parce que on mange et puis après la nuit on grandit, mais on le voit pas \\
\hline $\mathrm{M}:$ & $\begin{array}{l}\text { et pour les poules comment ça se passe pour que le petit poussin il devienne une } \\
\text { poule adulte }\end{array}$ \\
\hline Nina: & c'est comme nous / eh ben en fait il mange ça ça devient comme nous \\
\hline M : & $\begin{array}{l}\text { mais il mange pas de la poule alors comment ça se fait que ça devient de la } \\
\text { poule / qu'est-ce qui se passe dans son corps / comment il fait pour fabriquer des } \\
\text { plumes de la crête / qu'est-ce qu'elle peut faire la nourriture à ton avis }\end{array}$ \\
\hline
\end{tabular}

Nina : elle devient / de la peau 
Nina fait d'emblée le lien entre la croissance et la nutrition chez l'humain, mais les mécanismes qui permettent aux êtres vivants de grandir restent très flous (ils se passent la nuit et on ne le voit pas). Elle met ensuite en relation ce qui se passe chez les humains et ce qui se passe chez la poule ("c'est comme nous »). En disant que la nourriture devient de la peau, elle semble manifester l'idée de transformation de la matière, mais ce n'est pas encore formulé en ces termes.

Cependant, deux séances plus tard, quand Nina est amenée à produire un écrit pour expliquer comment les êtres vivants grandissent, on voit explicitement apparaître les idées de transformation de la nourriture et le fait qu'une partie ressort sous forme de déchets : "C'est la même chose pour la poule et l'enfant, d'abord il mange et après les déchets et il garde une partie du manger. Ça se transforme. Pour la plante, elle boit de l'eau et ça se transforme. " Il y a donc une avancée dans le mode de raisonnement de cette élève en faveur de la construction d'explications scientifiques relatives à la croissance. D'après nous, cette évolution trouve très probablement son origine dans les séances intermédiaires et en particulier lors une séance comportant un débat collectif dans la classe de Nina. Nous allons voir que les échanges oraux collectifs contribuent à enrichir les conceptions individuelles des élèves.

\subsubsection{Apport des échanges langagiers dans la recherche d'explications}

Les tableaux 6 et 7 présentent des extraits de la retranscription du débat collectif de la séance 4 . Nous parlons de débat scientifique au sens d'échanges oraux visant la recherche et la production d'explications scientifiques (voir Schneeberger \& Vérin, 2009) [22]. Les élèves viennent de réaliser une affiche par petits groupes. Le premier extrait correspond à la mise en commun des explications dans le cas de la croissance humaine.

Tableau 6. Extrait 1 : retranscription des échanges collectifs dans la classe de Nina $(M=$ maîtresse ; $\mathrm{A}^{*}, \mathrm{I}^{*}, \mathrm{Y}^{*}$ élèves)

\begin{tabular}{ll}
$\mathrm{M}:$ & $\mathrm{A}^{*}$ tu vas nous expliquer ce que vous avez dit tu te rappelles un peu \\
\hline $\mathrm{A}^{*}:$ & en ben quand on mange la nourriture \\
\hline $\mathrm{M}:$ & $\begin{array}{l}\text { quand on mange de la nourriture // alors qu'est-ce qui s'passe // I* tu veux } \\
\text { venir lire la phrase que vous avez faite / il va t'aider un peu }\end{array}$ \\
\hline $\mathrm{I}^{*}:$ & $\begin{array}{l}\text { (vient près de l'affiche et lit) on mange une partie de la nourriture sort / caca } \\
\text { et pipi... }\end{array}$ \\
\hline $\mathrm{M}:$ & $\begin{array}{l}\text { alors je relis parce que je sais pas si vous avez entendu on mange / une partie de } \\
\text { la nourriture sort c'est le caca et le pipi et une partie... }\end{array}$ \\
\hline $\mathrm{I}^{*}:$ & une partie se transforme en matière pour le corps \\
\hline $\mathrm{M}:$ & $\begin{array}{l}\text { et une partie se transforme en matière pour notre corps / ça veut dire qu'on se } \\
\text { servirait de la nourriture pour // }\end{array}$ \\
\hline Nina : & pour grandir \\
\hline $\mathrm{M}:$ & $\begin{array}{l}\text { ben oui pour fabriquer plus plus de matière plus de corps / d'accord et à force } \\
\text { d'avoir plus de matière on devient plus grand et plus épais d'accord }\end{array}$ \\
\hline $\mathrm{Y}^{*}:$ & comme toi
\end{tabular}

Les idées de transformation et de déchets à partir de la nourriture ingérée apparaissent explicitement dans les propos de l'élève I* (énoncés en gras). Or ce sont celles qu'on retrouve dans les explications de Nina. Il y a une évolution qui se produit chez cette élève à travers des déplacements de significations des termes comme "devient», "se transforme ». Le lien entre la croissance et la fabrication de nouvelle matière vivante à partir de la nourriture semble se mettre en place à travers un travail par le langage.

Par ailleurs, il est intéressant de remarquer que les élèves de cette classe sont engagés dans une recherche d'explications comme en témoigne le deuxième extrait qui suit. Les élèves poussent leur 
questionnement au delà de la question des mécanismes à l'origine de la croissance. Si manger sert à grandir, alors pourquoi la maîtresse qui ne grandit plus mange encore ?

Tableau 7. Extrait 2 : retranscription des échanges collectifs dans la classe de Nina $\left(M=\right.$ maîtresse ; $S^{*}, I^{*}$ élèves)

M : $\quad$ oui alors y en a qui m'ont posé la question dans ces deux groupes-là /, mais toi tu grandis plus alors pourquoi tu manges encore / c'est une bonne question

\begin{tabular}{ll}
\hline$S^{*}:$ & ben moi je sais \\
\hline$M:$ & oui $S^{*}$ \\
\hline$S^{*}:$ & ben sinon tu meurs \\
\hline$M:$ & eh oui parce que manger et boire \\
\hline$I^{*}:$ & c'est pas que pour grandir \\
\hline$S^{*}:$ & c'est pas exactement pour grandir \\
\hline$M$ & $\begin{array}{l}\text { c'est pas que c'est exactement pour grandir c'est comme dit } I^{*} \text { ce n'est pas que } \\
\text { pour grandir }\end{array}$ \\
\hline$S^{*}:$ & $\begin{array}{l}\text { oui parce que si si si c'était pour grandir si si moi je voulais pas grandir je } \\
\text { mange pas et je meurerai }\end{array}$ \\
\hline$M:$ & $\begin{array}{l}\text { je mourrai / oui donc après même si moi je ne grandis plus / quand on est adulte } \\
\text { on arrête de grandir }\end{array}$
\end{tabular}

Les interventions de l'élève $S^{*}$ (en gras) indiquent qu'elle envisage la nutrition comme un moyen de se maintenir en vie : on ne mange pas uniquement pour grandir, mais aussi pour ne pas mourir.

Finalement, cette deuxième séquence en CE1 amène les élèves au delà d'une simple accumulation des caractéristiques du vivant. Ils sont incités à produire des explications par des mises en relation (par exemple entre croissance et nutrition), une recherche de sens et cela contribue à l'enrichissement de la construction, déjà engagée, du concept de vivant. C'est pour nous un signe fort d'acculturation scientifique. L'exemple de Nina montre qu'au début du CP elle a une vision du vivant comme quelque chose qui bouge, qui a des caractéristiques semblables à l'Homme et sélectionne souvent une caractéristique pour un être vivant. A l'issue des activités menées au cours des deux années, Nina reconnaît désormais ce qui est vivant en fonction de caractéristiques du vivant reconnues par les scientifiques, elle réinvestit et combine ces caractéristiques, elle fait des liens entre les manifestations de la vie chez différents êtres vivants et elle semble s'être appropriée des pratiques spécifiques aux sciences telles que la production d'explications.

\section{Conclusion}

Toutes ces raisons nous conduisent à dire que ce sont des signes de développement du psychisme au sens où le décrit Brossard (2013) [20] :

«Le maître en lui proposant des tâches culturellement complexes et qui font sens à ses yeux - comme produire une explication par écrit - le conduit à mettre en œuvre des activités nouvelles et de ce fait à transformer son mode de fonctionnement habituel. C'est donc en fin de compte l'activité propre de l'élève suscitée par des tâches hautement exigeantes, qui est à la source du développement. » (p. 29-30)

Les processus d'acculturation scientifique que nous avons souhaité induire à l'école sont une forme de développement, car les élèves construisent un nouveau rapport au monde. Ils dépassent leur rapport quotidien et transforment peu à peu leurs connaissances pour accéder aux savoirs scientifiques relatifs au vivant. Cela fait écho aux propos de dell'Angelo (2009) [15] sur les enjeux sous-jacents à l'enseignement du vivant et s'ancre dans la problématique du colloque en mettant en lumière le défi que constitue l'enseignement-apprentissage du vivant en contexte scolaire. Les élèves de 5-7 ans, qui ont pris part aux deux séquences sur le vivant, sont engagés dans des processus de transformation de 
La « vie » et le « vivant » : de nouveaux défis à relever dans l'éducation

leurs connaissances quotidiennes pour aller vers une conception du vivant qui soit compatible avec les savoirs scientifiques actuels de la biologie et qui pose les bases d'une formation scientifique, à poursuivre au cours de leur scolarité ultérieure.

Deux remarques néanmoins quant aux processus d'acculturation scientifique. Premièrement, si les enseignants mettent en œuvre des situations favorables pour induire ces processus chez leurs élèves, ils ne peuvent pas les décréter. Ce sont bien les élèves qui s'y engagent et qui génèrent en eux-mêmes des reconfigurations dans leur façon de concevoir le monde. Deuxièmement, l'acculturation prend du temps et on ne peut envisager le développement humain qu'à long terme.

En définitive, nous avons étudié dans cet article des processus d'acculturation scientifique qui nous semblent rendre compte de la complexité à enseigner le vivant et qui sont l'occasion d'apprécier les effets d'apprentissages scientifiques sur le développement psychique des enfants. L'acculturation aux sciences ne se limite pas à l'acquisition de savoirs; elle passe aussi par l'appropriation de pratiques telles qu'argumenter. Cela suppose un changement de posture énonciative: mettre à l'épreuve ses idées, s'inscrire dans une communauté discursive scientifique à dimension scolaire au sein de laquelle les élèves peuvent construire des preuves acceptables par et pour le groupe classe. Les situations présentées font ressortir que l'acculturation se produit par juxtaposition de séquences abordant le même concept de façon différente. Les résultats obtenus dans cette étude montrent que les orientations retenues pour l'enseignement du vivant à destination d'élèves âgés de 5 à 7 ans semblent contribuer à la construction d'une conception scientifique du vivant et plus largement à leur entrée dans une première culture en biologie. D'autres situations pourraient être propices à compléter la construction du concept de vivant : citons les rencontres avec des êtres vivants dont les élevages que nous avons évoqués font partie (Charles, dans cet ouvrage) [26] ou encore des moments de réflexivité pour permettre aux élèves de prendre du recul sur les apprentissages qu'ils réalisent (Noack, dans cet ouvrage) [27].

\section{Références}

1. G. Bachelard, La formation de l'esprit scientifique: contribution à une psychanalyse de la connaissance objective (Librairie philosophique J. Vrin, 1980).

2. Ministère de l'Education Nationale, Programmes d'enseignement de l'école primaire, Bulletin officiel hors série $n^{\circ} 3$ du 19 juin 2008 (2008).

3. G. Kostyrka, Définir la vie en biologie : trois problèmes, In T. Hoquet \& F.Merlin (dir.) Précis de philosophie de la biologie (p.185-196), (Vuibert, Paris, 2014).

4. H. Bersini \& J. Reisse (dir.) Comment définir la vie? les réponses de la biologie, de l'intelligence artificielle et de la philosophie des sciences (Vuibert, Paris, 2007).

5. M. Morange, La principale difficulté pour une définition de la vie : concilier continuité et discontinuité, In H. Bersini \& J. Reisse (dir.), Comment définir la vie? les réponses de la biologie, de l'intelligence artificielle et de la philosophie des sciences (p.65-70) (Vuibert, Paris, 2007).

6. J. Guichard \& J. Deunff (dir.), Comprendre le vivant: la biologie à l'école (Hachette, Paris, 2001).

7. M. dell-Angelo, "Vie» et "vivant», perspectives épistémologiques, In La "vie » et le "vivant ": De nouveaux défis à relever dans l'éducation (2015).

8. C. Simard, L. Harvey \& G. Samson, Épistémologie de la biologie et conceptualisation du vivant chez des futurs enseignants et biologistes, In La «vie» et le "vivant»: De nouveaux défis à relever dans l'éducation (2015).

9. M. Coquidé, "Se sentir vivant»: quels enjeux d'éducation biologique, In La "vie» et le «vivant »: De nouveaux défis à relever dans l'éducation (2015).

10. C. Bardel \& E. Triquet, Grand N, 61, 87-104 (1997).

11. D. Nury, J. Lamarque \& P. Caron, Didaskalia, 9, 157-172 (1996).

12. M.-H. Parizeau, Comment la biologie répond-elle à la questions : qu'est-ce que la vie ?, In $L a$ « vie » et le « vivant » : De nouveaux défis à relever dans l'éducation (2015). 
13. F. Piron, Science et débat public sur le vivant, In La «vie» et le «vivant» : De nouveaux défis à relever dans l'éducation (2015).

14. M. dell'Angelo \& C. Roux-Goupil, Les valeurs associées aux enseignements relatifs au vivant, Communication présentée au Colloque de l'ACFAS La «vie » et le «vivant» : De nouveaux défis à relever dans l'éducation (mai 2015).

15. M. dell'Angelo-Sauvage, La construction d'un rapport au vivant: un autre regard sur les enseignements relatifs aux vivants à l'école et au collège (Delagrave, Paris, 2009).

16. J. S. Bruner, L'éducation, entrée dans la culture: les problèmes de l'école à la lumière de la psychologie culturelle (Retz, Paris, 1996).

17. L. S. Vygotski, Pensée et langage (La Dispute, Paris, 1934/1997).

18. R. Driver, H. Asoko, J. Leach, P. Scott \& E. Mortimer, Educational Researcher, 23(7), 5-12. (1994).

19. D. Cuche, La notion de culture dans les sciences sociales (La Découverte, Paris, 2010).

20. M. Brossard, Thématique 1 - première section, In M. Brossard \& J.-P Bernié, Vygotski et l'école : apports et limites d'un modèle théorique pour penser l'éducation et la formation (p. 21-34) (Presses universitaires de Bordeaux, Pessac, 2013).

21. M. Jaubert, Langage et construction de connaissances à l'école : un exemple en sciences (Presses universitaires de Bordeaux, Pessac, 2007).

22. P. Schneeberger \& A. Vérin (dir.), Développer des pratiques d'oral et d'écrit en sciences : quels enjeux pour quels apprentissages (Institut National de Recherche Pédagogique, Lyon, 2009).

23. C. Orange, Étude des situations "forcées" : quelles méthodes pour les recherches didactiques s'appuyant fortement sur les productions des élèves et de la classe ?, Actes du congrès de l'AREF (2010).

24. P. Paillé \& A. Mucchielli, Les analyses qualitatives en sciences humaines et sociales (Armand Colin, Paris, 2012).

25. G. Lecointre, Comprendre et enseigner la classification du vivant (Belin, Paris, 2004).

26. F. Charles, Les rencontres des enfants avec la vie et le vivant à l'école maternelle, In La «vie » et le " vivant»: De nouveaux défis à relever dans l'éducation (2015).

27. J. Noack, Apprendre, un moment de vie ? Savoir, expérience et rapport au vivant selon Georges Canguilhem, In La « vie » et le "vivant» : De nouveaux défis à relever dans l'éducation (2015). 